Illinois State University

ISU ReD: Research and eData

Theses and Dissertations

$4-10-2014$

\title{
When Can We Meet For Coffee?: Strategies In Online Dating To Build Liking When Exchanging Mediated Messages
}

Jacob I. Hazzard

Illinois State University, jhazz72@gmail.com

Follow this and additional works at: https://ir.library.illinoisstate.edu/etd

Part of the Communication Commons

\section{Recommended Citation}

Hazzard, Jacob I., "When Can We Meet For Coffee?: Strategies In Online Dating To Build Liking When Exchanging Mediated Messages" (2014). Theses and Dissertations. 128.

https://ir.library.illinoisstate.edu/etd/128

This Thesis is brought to you for free and open access by ISU ReD: Research and eData. It has been accepted for inclusion in Theses and Dissertations by an authorized administrator of ISU ReD: Research and eData. For more information, please contact ISUReD@ilstu.edu. 


\title{
WHEN CAN WE MEET FOR COFFEE?: STRATEGIES IN \\ ONLINE DATING TO BUILD LIKING WHEN \\ EXCHANGING MEDIATED MESSAGES
}

\author{
Jacob I. Hazzard
}

52 Pages

May 2014

This study investigates what strategies are implemented in online dating settings by online daters that lead to an offline meeting. The variables of openness and attentiveness were examined as the main strategies that lead to liking between individuals using this dating medium. The study compared levels of openness and attentiveness between groups of online daters who decided to meet offline to those who decided not to meet offline after exchanging messages on an online dating site. The results show there is a higher level of both openness and attentiveness reported by the participants who met someone offline when compared to an experience where they did not meet someone offline. Although these variables are not a predictor in whether the offline meeting will be successful, they seem to be predictors of getting to the offline meeting, which is considered a successful use of the online dating medium. The study also found that men were more likely to be the initiators to ask for an offline meeting when compared to women. 
WHEN CAN WE MEET FOR COFFEE?: STRATEGIES IN

ONLINE DATING TO BUILD LIKING WHEN

EXCHANGING MEDIATED MESSAGES

JACOB I. HAZZARD

A Thesis Submitted in Partial Fulfillment of the Requirements for the Degree of

MASTER OF SCIENCE

School of Communication

ILLINOIS STATE UNIVERSITY

2014 
Copyright 2014 Jacob I. Hazzard 
WHEN CAN WE MEET FOR COFFEE?: STRATEGIES IN

\section{ONLINE DATING TO BUILD LIKING WHEN}

EXCHANGING MEDIATED MESSAGES

JACOB I. HAZZARD

COMMITTEE MEMBERS:

William R. Cupach, Chair

John R. Baldwin

Kevin R. Meyer 


\section{ACKNOWLEDGEMENTS}

This thesis is dedicated to those that came before for me and those that will come after me. It is dedicated to those that stood by me and those that aided me in its completion. It has been a long and rewarding experience that will forever stay with me. More specifically I would like to thank Dr. Bill Cupach for sticking with me these years he was never willing to let anything compromise the integrity or the potential of this thesis. To Dr. Kevin Meyer for always making sure that I was presenting my information with statistical accuracy and for asking the questions that lead to this completion. To Dr. John Baldwin for always cutting any tension with his puns and sense of humor that helps a student like me stay motivated and not overwhelmed. And lastly, to my parents, Richard and Nicki Hazzard, and my aunt and uncle, Christy and Jack Meldrum, who provided me with constant encouragement and helped provide the means for me to complete my education. 


\section{CONTENTS}

Page

ACKNOWLEDGEMENTS CONTENTS CHAPTER

I. INTRODUCTION 1

II. REVIEW OF LITERATURE 5

Computer-mediated Communication $\quad 5$

$\begin{array}{ll}\text { Online Dating } & 8\end{array}$

History and Types $\quad 8$

Process 10

$\begin{array}{ll}\text { Liking Strategies } & 13\end{array}$

$\begin{array}{ll}\text { Openness } & 14\end{array}$

Attentiveness 18

$\begin{array}{ll}\text { Hypotheses } & 21\end{array}$

$\begin{array}{ll}\text { III. } & \text { METHOD }\end{array}$

$\begin{array}{ll}\text { Procedure } & 24\end{array}$

Participants 25

Measures 28

Data Analysis $\quad 29$

Factor Analysis $\quad 29$

Reliability 29

$\begin{array}{lll}\text { IV. } & \text { RESULTS } & 31\end{array}$

$\begin{array}{lll}\text { V. DISCUSSION } & 33\end{array}$ 
Limitations 


\section{CHAPTER I}

\section{INTRODUCTION}

One of the greatest wonders in most people's lives is searching for and discovering the person with whom they want to spend the rest of their lives. Romance occupies much of our time outside of work. Some are lucky enough to find the "right" person with their first try and some never find that certain someone, despite their search. Now, many people are turning to alternative dating methods due to changes in culture, such as busier work schedules (Henry-Waring \& Barraket, 2008). Although stigmatized, online dating has become a more popular venue to find a long-term romantic relationship (Ellin, 2009; Smith \& Duggan, 2013).

With the convenience of the Internet, millions have chosen to use online dating as an efficient and convenient way to meet potential romantic partners. It has been reported that about 16 million people use online dating sites (Madden \& Lenhart, 2006). One in ten Americans has reported using an online dating site or phone application and $66 \%$ of these daters say they have gone on a date with someone from the online dating site (Smith \& Duggan, 2013). Another report says 22\% of heterosexual couples and $61 \%$ of same-sex couples reported meeting online (Gardner, 2012). This new medium for relationship initiation has not replaced traditional means of seeking intimacy but rather coexists side by side with it (Sprecher \& Metts, 2013). In other words, the traditional means of meeting romantic partners still exist and thrive, but now online dating is used to cover more ground when searching for a romantic partner. The online dating industry 
itself was expected to top 1.049 billion dollars in 2009 and grow at a rate of $10 \%$ each year (Mitchell, 2009). This is a large industry that is continually growing in revenue accrued and in number of users. Based on this fact and because online dating changes how we approach seeking relationships, it is easy to see the importance of researching how online dating functions, how it is used, and how it differs from more traditional means of dating.

Despite being called "online" dating, the actual dating does not occur online. Rather, sites are used as a way to initiate contact that leads to an offline, face-to-face (FtF) meeting where most of the actual traditional dating occurs (Long, 2010; Sprecher, 2011). A more appropriate term would be online initiating, but since online dating is the most commonly used phrase, the present study will continue to utilize it as a reference to the interactions that occur within websites that promote relationship initiation. Although there are many places to meet new people online such as chat rooms or interest group sites (McKenna, 2007), this study will be focused on websites that are designed specifically for meeting others online to initiate dates. These particular sites are referred to as targeted websites because they are designed to meet romantic partners as opposed to other types of relationships (McKenna, 2008). It should be noted that there are relationships that form and stay online (Rabby, 2007); however, for this study, the focus is on persons who use websites to initiate relationships, and then move to a FtF meeting.

There is an abundance of research concerning online dating and how it relates to communication. Most of the research revolves around impression management (Ellison, Heino, \& Gibbs, 2006), which includes deception in user profiles (Guadagno, Okdie, \& Kruse, 2012). There are also studies of who uses online dating (Valkenburg \& Peter, 
2007) and why people decide to join an online dating site (Whitty, 2008). Recently there has been more of a focus on how the process of online dating functions. In a comprehensive report about online dating, Finkel, Eastwick, Karney, Reis, and Sprecher (2012) lay out a nine-step process, taken in part from Long's (2010) study, as to how the majority of online relationships are developed. The current study will seek to broaden and expand the step in which mediated messages are exchanged.

Although Finkel et al. (2012) argue that the online dating process is relatively new, there are certain aspects that are the same as the traditional dating process, if only extended. The step of getting to know each other to decide to meet for a private, one-onone date is typically accomplished by hanging out in a social group (Sprecher \& Metts, 2013) or via short conversations in a social scene where numbers are exchanged, such as at a singles bar. There must be some factors that determine why a person who exchanges messages with another potential online dater agrees to move to a FtF meeting, just like when a person agrees to exchange numbers or meet for a private date in traditional settings.

The purpose of this study is to explore whether or not the processes that increase liking that apply in a traditional dating setting to initiate relationships are applicable in a newer medium, online dating. Online dating is more of a relationship initiation place, and generally the dating takes place in offline meetings. Liking is an important concept when one is establishing a relationship and can determine whether or not two people decide to continue to develop a new relationship (Hendrick \& Hendrick, 1992). The more liking is generated while initiating relationships online, then the more likely there will be success in moving to an offline meeting. 
There are specific strategies that are important to increasing liking in a traditional, offline meeting. This study hopes to determine whether these strategies apply online as well in that they aid a couple in moving to an offline meeting. To better understand the current study we must first understand the background of communication in a computermediated conversation and examine what online dating is and who does it. 


\section{CHAPTER II \\ REVIEW OF LITERATURE \\ Computer-Mediated Communication}

Because the initial interaction between two people who utilize online dating sites takes place in an online environment, it is a form of computer-mediated communication (CMC). Any time that a computer is used in place of a FtF conversation, it is commonly referred to as CMC. A "computer" in this case could be seen as a device that has a computer chip in it which includes, but is not limited to, tablets, cell phones, and laptops.

Because CMC environments are typically text based, there tends to be little-to-no nonverbal cues, such as facial expression, as well as reduced contextual and auditory cues, such as sarcasm. Due to these cues being filtered out by the medium being used, many scholars believed that people would not want to or would not be able to form meaningful relationships through CMC (Rice \& Love, 1987). Cues filtered out scholars felt that without these cues people would have a reduced experience and not be able to have "real" interaction that typically leads to relationship formation (Finkel et al., 2012). If this were true, then people using online dating would find much less success than they do currently; they would more than likely not be able to progress to a FtF meeting.

Walther (1992) challenged the cues filtered out paradigm, which led to the development of the Social Information Processing Theory (SIPT). Walther found that if the time frame was eliminated from interactions, then people would tend to form relationships just as they do in FtF interactions (Walther, 1992, 1996). Basically, people 
are social creatures and will use any tool to be socially interactive. They will still form similar relationships as $\mathrm{FtF}$, but because of the medium and its lack of cues, it becomes a longer process than FtF because less information can be conveyed to a conversation partner with each message.

SIPT became a viable lens to examine online dating, and scholars began to believe that romantic relationships can be formed using CMC when given enough time (Merkle \& Richardson, 2000). Although SIPT explains that satisfying relationships can be formed, it does not perfectly explain all that happens with relationships in online dating sites. This is mainly due to the fact that online dating sites are used to initiate relationships, but such relationships do not develop fully in the online environment. Much of the relationship is formed after the two users meet FtF. SIPT is most useful in explaining relationships that form completely online and is only able to explain a small part of those that are initiated online and then move to FtF (Long, 2010).

Walther (1996) expanded his research to introduce the concept of the hyperpersonal model in CMC, which is an extension of SIPT. This model provides a framework explaining the transformations of relational communication and participants through online interactions (Walther, 1996). In other words, this model explains how CMC users are able to showcase themselves and how they are able to control their selfpresentations online. People try to put their best face forward in CMC by engaging in selective self-presentation (O’Sullivan, 2000). For example, in text-based CMC such as instant messaging, people may enhance their impression management through careful and thoughtful message composition. There are many activities, such as with clothing or make-up, that offline daters can use to control their self-presentation; however, online 
dating affords many unique opportunities for selective self-presentation in profile creation that are not available in FtF meetings.

Online, the individuals can upload a picture that omits any features they do not like about themselves, thus leading to a picture that exemplifies how the individual wants to be seen. Related to the sender's behavior in CMC, individuals appear to be more comfortable disclosing information about themselves in $\mathrm{CMC}$ rather than $\mathrm{FtF}$ (Walther, 2007). In addition, senders tend to highlight their positive traits in order to lessen their negative traits (Ramirez \& Zhang, 2007).

The hyperpersonal model explains why people meeting FtF after initiating on online dating sites have more information about their prospective partners before they meet. This pre-meeting information is something different between a FtF meeting after initiating online and meeting FtF after initiating offline. The hyperpersonal model explains how when communicating online, users tend to create idealized models of conversation partners. The users then tend to search out more information to verify whether or not that person matches with that view. Because of the amount of selfpresentation people use when constructing the online profile, much of the information about a person is available to anyone who visits the profile (Long, 2010). Despite explaining where and how people obtain information on each other, the hyperpersonal model does little to explain how people interact on the site or after they decide to move offline, which is something this study looks to expand.

Online dating presents a unique challenge to explain current relationship initiation. Because such initiation begins with the partners having a wealth of previous knowledge about each other before they meet, many of the traditional offline theories of 
relationship formation do not apply. SIPT and the hyperpersonal model explain certain aspects of the online dating process but not all of them. Now that we better understand the medium in which online dating operates, it will be beneficial to understand the history of online dating and how it functions.

\section{Online Dating}

\section{History and Types}

It was not until the emergence of high-speed Internet and personal, home computers that online dating as a viable option began to thrive. The first widely recognized website was Match.com, which started in 1995 (Sprecher, 2011; Sprecher \& Metts, 2013). This introduced the idea that one could now look anywhere in the world for a potential match. Match.com is primarily a site that is used like a personal ad section that was in newspapers of the past. These types of sites are known as self-selection sites because the user determines who he or she thinks will be a good match with no compatibility screening from the website in choosing whom they select; however, these sites tend provide a space that is more accessible to a greater number of people and can go into more depth than personal ads because generally there is no limit to what a user can put in his or her personal profile, unlike a personal ad (Long, 2010).

In 2000, sites began to use scientific matching as part of their service. eHarmony was one of the first widely known sites that offered a way to match online daters to other users based on responses to specific questions or through the use of lengthy questionnaires that the site has subscribers complete. These questions can measure anything from personality types to one's "love style" (Long, 2010). The matching sites claim to match through algorithms that are based on social science research. Many sites 
claim these algorithms sift through responses to match users with other users who may display similar, compatible, or complementary interests (Orenstein, 2003). Despite their claim to be developed in science, most sites have not released their matching formulas to be peer reviewed, so it is difficult to determine their validity (Sprecher \& Metts, 2013). To their credit, many of the larger sites have hired or consulted known social scientists to help them develop their algorithms and analyze any data the sites may collect on their users (Finkel et al., 2012). A few more sites that utilize this strategy are chemistry.com and perfectmatch.com. These sites tend to allow users to create and view profiles of others at no cost, but when it comes to actually contacting others, they begin to charge a fee for the service.

Many sites, both self-selection and matching, do not charge for their services and get revenues from advertisements or from upgraded services. Some of the more popular of these free sites are pof.com, known as plenty of fish, and OkCupid.com. There are also many sites that are tailored to specific sub-groups both in self-selection and matching varieties. These could be based on religion, such as Judaism (Jdate.com) or Christianity (christiansingles.com), political view (liberalhearts.com), age group (seniorpeoplemeet.com), or sexual orientation (Gay.com). These tailored sites tend to be niche sites that have fewer members, but cater to those who have a particular trait that stands out for them (Sprecher \& Metts, 2013). There are also sites that are developed for those searching just for "hook-ups" or short term romances, such as adultfriendfinder.com. However, in the current study the focus will be on sites that promote longer-term relationships.

People have always used new technologies, such as video dating or want ads (see 
Ahuvia \& Adelman, 1992), to meet potential partners. Online dating may just be an extension of that similar behavior in using new technology to find dates. However, online dating seems to be more pervasive than past technologies and more widely accepted by society as whole than that of want ads and video dating (Finkel et al., 2012), which could be due to the higher accessibility and the increased space to describe oneself.

\section{Process}

The steps an individual must take when utilizing online dating are important to understanding the current study. The process starts with what goes into choosing to use an online dating site, and ends with what happens when two people that initiated contact online move their relationship to a FtF meeting. This process was detailed in Long's (2010) study as a nine-step process. The first step is gathering information about online dating in general or about specific sites one might be considering. There are many reasons why a person may turn to online dating such as relocation to a new area, a recent break up, problems finding partners, or just curiosity (Finkel et al., 2012).

Once the user has decided on a site to join, he or she must go through the registration process, which can bear a financial cost depending on which site is chosen. The third step is where the user actually creates a profile. A profile is the webpage that other users can view and use to make assessments on whether or not a user is a potential partner. This is similar to personal ads of the past, but with much greater detail and fewer limitations as to the ways in which users can construct a synopsis of themselves. This is where online dating tends to diverge from traditional dating, because this user-created profile does not exist in traditional settings (Finkel et al., 2012). Although daters can search for a person via social networking sites and search engines (Gibbs, Ellison, \& Lai, 
2011), the online dating site presents information about users in an easily accessible format. The information presented varies from site to site, but generally has similar functions. Typically, these profiles contain at least one picture, if not more, of the user. Often, information is not required on the profile in either self-selection sites or matching sites; however, some sites require certain questions to be answered to aid in the matching. Even if the user has an abundant amount of information in his or her profile, without a picture, others will likely pass over their profile (Fiore, Taylor, Mendelsohn, \& Hearst, 2008). The profile also contains general, demographic information such as age, sex, religion, height, weight or body type, and so on. The information that is included in a profile is completely in control of each user.

The next step is optional, but is still done by most users. This step involves viewing other users' created profiles. This is normally done through a search function provided by the website. The search function differs for each individual site in varying degrees on how the user can focus his or her search for others. The user generally has an idea of what he or she is looking for in different aspects of a potential partner such as proximity and perhaps physical traits. Because users have an idea or agenda when approaching this search, much like a shopping list, some researchers term this process as “relation-shopping” (Heino, Ellison, \& Gibbs, 2010; Whitty \& Carr, 2006). Notably, some sites match users to other users by what the site determines would be a good match based on self-report items (e.g., eHarmony), whereas other sites leave the selection process strictly up to the users to browse others' profiles to determine a match (e.g., Match, OKCupid).

The fifth and sixth steps are related in that they are the actual sending and 
receiving of initial messages. Some users never send a message to another user and simply rely on others to initiate interactions, thus skipping a step. As in traditional dating, men tend to initiate more relationships than women, and thus send more initial messages than they receive (Hitsch, Hortaçsu, \& Ariely, 2010).

The seventh step is the most crucial to the current study. It involves the exchange of messages via mediated means. This can be done in many different ways, including using the messaging options from the site, exchanging and using personal emails, or even exchanging phone numbers and communicating via text or voice; thus, this is when liking strategies are used to move to the FtF interaction. This step is crucial in getting to a FtF meeting, which is considered one measure of success when using an online dating site. If enough liking for one another develops, then eventually the pair will meet offline. This typically occurs within a month of first contact and even sometimes a week (Whitty, 2008).

The actual FtF meeting is the eighth step of the online dating process. At this point the site tends to become irrelevant and the budding relationship tends to be akin to traditional dating (Whitty \& Carr, 2006). This first date is treated as a screening process that allows the users to determine how much the person is like their projected online self (Long, 2010). The user will also be able to see if there is "chemistry" between self and the other person. If successful, this date leads to the final step, which is developing the offline relationship. At this point the process looks like traditional dating after a first date and progresses in a similar fashion. Often, if the relationship is successful the two users will leave the dating site or deactivate their profile (Long, 2010). 


\section{Liking Strategies}

Liking is difficult to define because often we just know that we "like" someone. Rubin (1973) describes liking as having two dimensions: affection and respect. Respect is liking that is based on personal accomplishments and characteristics of another person, whereas affection is liking based a personal connection or relationship with that person and can be felt through closeness (Rubin, 1973). Liking another person can lead to an increase of attraction to that person and can further the development of a relationship (Newcomb, 1961). Additionally, liking can vary in degree in that one can like one person more than another. Without liking there is little chance for a personal relationship to develop (Rubin, 1973). A dyad must use strategies to build liking in order to progress to a more intimate relationship, in this case an offline meeting.

No matter what type of initial encounter two people have, casual or romantic, in order for that encounter to be successful, rapport must be built (Bredow, Cate, \& Huston, 2008). The more rapport two people develop in an initial encounter, the more liking one will have for the other person. This holds true when initiating relationships online. The opening line occurs when one user finds someone she or he is interested in and sends a message. Just like traditional encounters, if the contacted person responds positively, then the encounter can progress to building liking. This is done online via messages on the dating website, or if the users have exchanged phone numbers, via text message and phone conversations. Strategies have been identified in traditional, offline relationships that are among the most effective in building liking, which are openness and attentiveness (Clark, Shaver, \& Abrahams, 1999; Hess, Fannin, \& Pollom, 2007). Relationships are doomed not to develop if there is no liking between parties (Rubin, 1973). 
To start any relationship there must be an initial meeting or some form of initiation. With online dating relationships, this initiation takes place on the online dating website. The initiation interaction that normally occurs when two people meet in any setting is extended in the mediated message exchange step because the communication is generally asynchronous. Due to the asynchronous communication the "initial meeting" is a longer process than in the traditional setting, which is explained by Walther's (1992, 1996) SIPT. Because online dating functions similarly to the initial meeting in offline relationships, the same strategies that are successful in the traditional settings should still apply in the mediated message exchange step in online dating, though the steps will be spread out across the exchanged messages.

\section{Openness}

Openness is defined in this study as the general process of communicating one's thoughts, feelings, ideas, and opinions with a potential partner. Bell and Daly (1984) discuss strategies that people use to gain liking and call it affinity-seeking strategies. They identified many of the ways that people seek to gain affinity. One of their defined strategies is labeled openness, which they define as when "the affinity-seeker discloses personal information to the target" (p. 97). Although they are called the same name and are related to the current study's definition of openness, there is some difference.

Although their strategies were developed to describe FtF meetings, openness can also be utilized in the mediated messages exchanged between two online daters. Another term that strongly overlaps with openness is self-disclosure. This study uses openness instead of self-disclosure because openness is a general disclosing of information, whereas selfdisclosure is often a more focused definition that refers to risky revelation of private 
information.

In initial meetings, each party uses information that is exchanged between them to determine the potential of a future relationship. This exchange is also what determines the amount of liking each has for the other (Derlega, Metts, Petronio, \& Margulis, 1993). The more we know about each other, the better we can make judgments on whether or not we wish to continue forming a relationship. In online dating, this informaion gain can occur uniquely during the step where a profile of another user is examined. There is a large amount of information about a person found in his or her profile because users construct this profile to represent themselves (Long, 2010). Openness occurs during the mediated communication phase, which can lead to a move offline if enough liking is generated between the users. If the users do not generate enough liking, they will generally cease communication and move on to their next prospect.

Surprisingly, those interacting online tend to reveal more about themselves more quickly than in FtF interactions (Merkle \& Richardson, 2000; Wysocki, 1998). Some of the increase in self-disclosure on CMC can be explained by the anonymity CMC provides because there is less fear of judgment from the other party (Henderson \& Gilding, 2004). Having long-term relationship goals can influence the disclosure that is used as well. Gibbs, Ellison, and Heino (2006) found that those using online dating with long-term dating goals are more honest with their disclosure, disclose more, and make conscious decisions about what they disclose. Having these goals then provides more openness within the message exchange with a potential partner and can lead to more liking.

Long-term dating goals can also be called intimacy goals. The more a person has intimacy as a goal when initiating a relationship, the more self-disclosure she or he will 
use to foster a more open conversation (Sanderson, Keiter, Miles, \& Yopyk, 2007). In Falk and Wagner's (1985) study, participants were given open-ended statements to disclose to a confederate. Some participants were given low-intimacy disclosure statements and some were given high-intimacy disclosure statements. The authors found that there is a higher probability for a relationship to develop as intimacy increased. Intimacy and intimacy goals can be expressed through openness between partners. With online dating, there is the concern amongst users that disclosure can be deceptive or misrepresent the person who is disclosing (Gibbs et al., 2006; Kang \& Hoffman, 2004). Intimacy goals in online dating will influence how honest or deceptive a person will be with their online profile. The more a person is looking for a serious relationship, the more honest they will likely be in their profiles and messages because that type of behavior leads to better success (Baker, 2005; Whitty, 2008).

Throughout the online dating process there are many opportunities to manage what information about a user is seen by other users, especially in profile construction and the exchange of mediated messages. This is done by highlighting positive traits in order to distract from negative traits (Ramirez \& Zhang, 2007). People will use technology media to manage their identities in this way (O'Sullivan, 2000). It may be easier to manage an identity with a profile on $\mathrm{CMC}$ than it is in a $\mathrm{FtF}$ interaction because responses can be thought out and constructed instead of just reacting to the other person in $\mathrm{FtF}$ interactions. Also, this medium reduces much of the interactions to text, which is easier to manage when comparing to $\mathrm{FtF}$ interactions where one has many more factors to control for. The text does not, however, have a temporal limit on the length of which the text can be studied. So, this allows more unique strategies for self-disclosure because 
online daters have more choice on what they select to present to their partners when picking out which pictures to show, and what information to put in their profiles as well as more time to study messages sent and received.

There are many links between liking and self-disclosure. Newcomb (1961) showed that similarity is a powerful predictor in attraction and thus liking. There are many reasons why people like those who are similar to themselves. There are many rewards for having a person who is similar to oneself such as easier communication, support for one's worldview, promotion of positive self-concept, and fostering anticipation for future interactions (Hendrick \& Hendrick, 1992; Rubin, 1973). Pinel, Long, Landau, Alexander, and Pyszczynski (2006) found that finding similar interests will lead to greater liking of the other individual. Sprecher, Treger, and Wondra (2013) separated participants in their study into listeners and disclosers in initial meetings to see how these roles influenced liking. They found that those receiving disclosure showed higher amounts of liking compared to those disclosing because of a perceived similarity with the discloser. This adds credence to the importance of being open in generating liking in online dating.

Collins and Miller (1994) did a meta-analysis of studies related to liking and selfdisclosure to further examine the link between these two variables. They concluded that there is a positive relationship between amount of disclosure and liking, both from disclosing to someone and being disclosed to. Liking also regulates self-disclosure in first encounters in that the more liking we have for a person the more we will disclose to them (Jourard, 1964). This can be affected by what Jourard and Landsman (1960) called the dyadic effect. The dyadic effect is the idea that people will disclose more to those who 
have disclosed to them; in other words, disclosure begets disclosure. So, not only will disclosing lead to more disclosure from another, it will also increase liking because more disclosure increases liking (Collins \& Miller, 1994). These concepts apply in online dating because not revealing enough to the other party so they cannot judge whether or not a first date would be worth their time would inhibit liking. Also, revealing more about oneself will lead to liking and more disclosure from the other party, which will in turn increase one's own liking for the other party. By being open with the other party, people will be more likely to like another person. In the current study, the focus will be on being open during the exchange of mediated messages.

\section{Attentiveness}

While it is important to reveal information about oneself, it is equally important to focus on the information that the other person is disclosing. This leads us to the second variable to generate liking: attentiveness. Much like openness, attentiveness is not easy to define simply because there are many terms that researchers use to refer to the same concept. For purposes of this study, attentiveness is defined as giving closer consideration to what is being disclosed by a conversation partner and utilizing that information to further a relationship. Attentiveness can be manifested in many ways such as showing respect to the other person, analyzing their messages and appropriately responding, and referring to previously disclosed information.

A similar construct that manifests the current definition of attentiveness is altercentrism, which can be defined as "attentiveness to what [the other person] says and how they say it, perceptiveness not only of what is said but also what is not said, responsiveness to their thoughts, and adaptation during conversation" (Rubin \& Martin, 
1994, p. 36). The current definition of attentiveness also manifests in the construct of interaction involvement (Cegala, 1984; Cegala, Savage, Brunner, \& Conrad, 1982). Interaction involvement examines the extent to which an individual is involved in a conversation with another person (Cegala et al., 1982). Cegala et al. discovered two dimensions within interaction involvement: perceptiveness, which is defined as "the extent to which one is knowledgeable of the meanings one ought to assign to other's behavior" (p. 230) and attentiveness, which is "the extent to which an individual is cognizant of stimuli that comprise the immediate environment" (p. 230). Both of these dimensions relate more closely to the current author's definition of attentiveness, than does Cegala et al.'s definition of attentiveness. Their definition would include knowing what meanings should be applied to other's behaviors and heeding cues from the other person (Cegala, 1984). This attention and focus on the other person will enhance liking that is being established in the initiation of the relationship. The more a person is perceived as focusing on others and the interaction, the more competent they appear to be at communicating, which makes them more likable due to ease of the interaction (Cegala, 1981).

In traditional dating environments, attentiveness can be displayed through physical cues, such as leaning towards the other or looking at their face, as well as nonsomatic ways, such as referring to past conversations (Norton \& Pettigrew, 1979). Because in online dating there are no physical cues, there is no way to gauge the physical indications of attentiveness. There are still many things an online dater can do to show attentiveness. This can include both acting respectfully towards the other person in the messages one sends and referring back to past messages the other person has sent. 
In online dating message exchange, displaying attentiveness via messages seems easier to accomplish than in traditional dating because the messages are typically saved by the website and are not spontaneous. This allows a person to review what information has been exchanged between the two. Also, there is more time to construct and review what is to be sent to the other person. However, communicators would not want to lose attentiveness by waiting too long to send a message. Constructing well thought-out messages shows orientation towards the other person because it displays that there has been time and energy dedicated to the message exchange. In a dating situation, this attentiveness shows the potential date that a person wants to further the relationship and is taking the communication and person seriously, which can ultimately lead to a FtF meeting. Without making the other person in an interaction feel important via displaying attentiveness to their communication, liking is doubtful (Spitzberg \& Dillard, 2002). Bell and Daly's (1984) affinity-seeking strategies are applicable to attentiveness as well. Their strategy of listening is very closely related to the current definition of attentiveness. The authors give an example of the listening strategy as "the affinity seeker asks the target for frequent clarification and elaboration, and verbally recalls things the target has said" ( $p$. 97). Although online message exchange is through a mediated channel, listening in this case would be paying close attention to what the other party has written and responding appropriately.

Openness and attentiveness are not the only factors that motivate two people to further their online relationship development by moving to a FtF meeting, but this study hopes to show that they are integral in developing enough of a connection online that will help propel two online daters to that FtF meeting. It is important for a user both to 
disclose to a potential partner while simultaneously showing the potential partner intended interest by being attentive to what is disclosed. In successful ventures of this online venue, both of these strategies are predicted to play an integral part.

\section{Hypotheses}

There are many behaviors that have been identified as ways to build liking in initial encounters or developing relationships. Because online dating, and more specifically the step in the process of online dating where the two individuals engage in mediated communication, is similar to a initiating an offline relationship (Finkel et al., 2012), these strategies can be applied to this new medium. Among the most prominent of these strategies are openness and attentiveness (Clark et al., 1999; Hess et al., 2007). Self-disclosure leads to more disclosure from the other party and more liking as a relationship develops (Altman \& Taylor, 1973). Attentiveness is linked to liking in that if the other person does not feel like they are important due to a lack of attention, there is a decrease in liking. But if the attention is given to the other person, they will feel important and increase the liking they feel (Spitzberg \& Dillard, 2002).

Online dating relationships represent a newly forming relationship that eventually moves offline. In most initial encounters both online and off, liking must be gained for a budding relationship to progress to a deeper level. In traditional dating, this initial encounter may occur at a public place such as a bar or coffee shop, or it may occur when

a group of friends all "hang out" (Bredow et al., 2008). These encounters typically do not last a long time and occur before a "first date." This initial interaction is comparable to the mediated message exchange in online dating. The difference is that this interaction 
online typically lasts on average a week to a month (Whitty, 2008) before participants move to the "first date" or first FtF meeting. In traditional dating, generally there is an agreed-upon meeting just between the two potential partners. SIPT explains why interactions online can still form relationships, but the time frame is longer than developing relationships offline (Walther, 1992; 1996). This seems to fit with why exchanging messages via online dating sites acts like an initial encounter, despite being stretched out for a week.

This study will examine whether some of the same strategies that have been used to build liking in initial offline relationships work in the same way as building liking in initial online relationships. The relationships that do move to an offline meeting will have used both openness and attentiveness to reach the meeting. Through the use of these two strategies, the two parties should have generated enough liking for each other that it leads to attraction, which then will promote them moving to a FtF meeting. Because liking has increased, there will be a relation to the likelihood and the speed in which online daters move offline. This leads to the first set of hypotheses:

$\mathrm{H}_{1}$ : Online daters who choose to further a developing online relationship by moving to an offline meeting will have a higher amount of openness during the mediated message exchange than those who choose not to move offline.

$\mathrm{H}_{2}$ : There is an inverse relationship between the amount of openness during the mediated message exchange and the time it takes online daters to move offline after the first message is exchanged. 
$\mathrm{H}_{3}$ : Online daters who choose to further a developing online relationship by moving to an offline meeting will have a higher amount of attentiveness during the mediated message exchange than those who choose not to move offline.

$\mathrm{H}_{4}$ : There is an inverse relationship between attentiveness during the exchange of mediated messages and the time it takes online daters to move offline after the first message exchanged.

In terms of liking-building strategies, it seems that men are much more likely to use more active and direct strategies in developing relationships and women tend to be more passive and use indirect strategies (Clark et al., 1999). This falls in line with the idea that men tend to send more messages than women in online dating site interaction (Hitsch et al., 2010). Women have also been known as the gatekeepers when it comes to allowing a sexual relationship (Cunningham, 1989) and therefore, may be more reluctant to move offline as quickly as men. This study proposes that it will also follow that once an exchange of messages has been started online, men will continue to be direct and attempt to move offline quicker than women. This leads to the fifth hypothesis:

$\mathrm{H}_{5}$ : Men will first suggest the move offline in a developing online dating relationship more often than women. 


\section{CHAPTER III}

\section{METHOD}

\section{Procedure}

The participants completed an online survey. First, they were given disclosures about the survey and were asked screening questions about their online dating experience. It was assumed they had experience interacting with others if they had used online dating sites. What was unknown was whether they had met someone offline subsequent to interacting on the online dating website. The screening question asked, "Have you ever exchanged messages with anyone on an online dating site (OKcupid, match.com, eHarmony, Plenty of fish, Zoosk, SinglesNet, or many others) that you eventually met offline?" This allowed the survey to direct the participants to either the first part of the survey, for those who had met someone, or to the second part of the survey, for those who had not met someone. Because many people who had met someone offline had also had experiences where they decided not to meet a different person offline, the second part of the survey was also completed by many of the participants who were first directed to the first part of the survey. Participants who had not had any online message exchanges were screened out of the survey.

The participants read a series of statements about their interaction with the other person. They then ranked their responses on seven point, Likert-type scales designed to measure openness and attentiveness during the period where messages were exchanged online. Participants were also asked many descriptive questions about their general 
experiences with online dating such as how long it took them to move offline, the extent of their experience with online dating, how long it had been since their interaction had taken place, as well as others. Another question, "who first asked to meet offline?" ("I did" or "The other person did"), helped determine which person asked to move the relationship offline.

\section{Participants}

Participants were 227 adults including 63 males (27.8\%) and 83 females (36.6\%). There were also 81 participants (35.7\%) who did not report their sex. The average age of participants was $27.99(S D=7.20$, range: $18-60)$. The average age of the other person who was met offline was $28.05(S D=6.86$, range: $18-66)$ and the average age of the other person not met offline was 27.68 ( $S D=6.57$, range: $19-61)$. In regards to racial demographics, 81 (35.7\%) participants did not report their race. However, $132(58.1 \%)$ reported being Caucasian, 2 (0.9\%) reported being African American, 3 (1.3\%) reported being Asian and Latino, respectively, $1(0.4 \%)$ reported being Pacific Islander, and 4 (1.8\%) reported as other. Also, one participant $(0.4 \%)$ chose not to disclose race.

The survey contained a section that was for participants who had had an interaction with someone online and then moved offline for a FtF meeting. The second part of the survey contained a section that applied to participants who had had an online interaction with someone and decided not to meet them offline. The number of participants who only answered the first part of the survey was $85(37.4 \%)$. The number of participants who took only the second section of the survey was $25(11.0 \%)$. The number of participants who completed both sections was 117 (51.5\%).

The participants were able to select from a list one or more dating websites with 
which they had experience in general. They also had the option to write in other websites they had utilized. These responses were not mutually exclusive nor did they represent the site they were using when they described relationships throughout the survey. Of the participants, 116 (51.1\%) reported using OkCupid, 42 (18.5\%) reported using Match, 24 (10.6\%) reported using eHarmony, 11 (4.8\%) reported using Zoosk, $2(0.9 \%)$ reported using Singlesnet, 47 (20.7\%) reported using Plenty of Fish. Within this sample, 25 participants (11.0\%) wrote in other sites with Tinder (5 participants) being listed the most often.

Data were captured that expressed the experience each participant had with online dating. Within the sample, 10 participants (4.4\%) reported having less than a month of online dating experience, 43 participants (18.9\%) reported having 1-6 months experience with online dating, 28 (12.3\%) reported having 6-12 months experience with online dating, and 65 (28.6\%) reported having more than a year of experience with online dating. Another 81 (35.7\%) participants did not report their experience with online dating or complete the section for this part of the survey.

The participants also described the time that had passed since the interaction took place with the other party whom they did not meet offline. Thirty (13.2\%) reported that the interaction was less than a month ago, $51(22.5 \%)$ reported that the interaction occurred one to six months ago, and another $30(13.2 \%)$ reported that the interaction occurred 6-12 months ago. Twenty-five (11.0\%) reported that their interaction was more than a year ago. In addition, 91 (40.1\%) did not report when their interaction took place or complete the section for this part of the survey.

The time that had passed since the interaction was also reported by the 
participants who did meet someone offline. This was only reported by those who had an experience where they met someone offline. Of those, $35(17.3 \%)$ reported that the interaction took place less than a month ago, 59 (29.2\%) reported that the interaction took place 1-6 months ago, $40(19.8 \%)$ reported that the interaction took place 6-12 months ago, and $31(15.3 \%)$ reported that the interaction took place more than a year ago. Thirtyseven $(18.3 \%)$ did not report how long ago the interaction took place. Participants also described whether or not they continued to date the person they met with $121(59.9 \%)$ reporting that they continued to date the other party with an average length of time of 7.64 weeks $(S D=11.13$, range $1-60)$.

The frequency in which participants checked their online dating site was reported. Within this group, $71(31.3 \%)$ reported that they checked the site more than once a day, $49(21.6 \%)$ reported that they checked once per day, 27 (11.9\%) reported checking every few days, 4 (1.8\%) reported checking once per week, and $13(5.7 \%)$ reported checking less often than once per week. Sixty-two participants (27.3\%) did not report how often they checked the online dating site or complete the section for this part of the survey.

The way this sample was gathered was a combination of a snowball sample and volunteers from forum websites. The participants were recruited via two separate methods. The first method was utilizing the author's social networking site, Facebook, to post the e-survey link online where participants had the option to voluntarily click the link and take the survey or pass the link along to others known to use online dating. The second method used the community forum website Reddit to post the link. The specific subreddits used for this were r/okcupid, r/samplesize, and r/ilstu. The subreddit r/okcupid was chosen because it relates to the subject and is the largest online dating community on 
Reddit based on the listed number of users who subscribe to this subreddit. The subreddit r/samplesize was chosen because it is a community of users who take and post surveys for research. Finally, r/ilstu was chosen because it is the community for the author's institution and had more willing participants. With both methods, participants were encouraged to share this study with others for whom it is relevant within their own social networks.

\section{Measures}

The items for both openness and attentiveness came from two sources. First, seven total items were borrowed and modified from Hess et al.'s (2007) Closeness Strategies Index (CSI). The CSI was created to measure offline interactions, so the modifications to the items were to adapt them for use in a mediated environment. Hess et al. (2007) labeled their variables as openness and attention, but the current author felt the items used for attention also described the author's definition of attentiveness. The rest of the items were created by the author of this study to increase reliability and strength of the scales.

All of the items for both openness and attentiveness were measured on seven point, Likert-type scales. There are a total of nine items that were used to measure openness. Three items are from the CSI (Hess et al., 2007). One sample item from the CSI (Hess et al., 2007) for openness was, "I was willing to discuss topics with this person that I wouldn't normally talk about with others online." Six of the items were created by the author for this study. A sample item for openness created by the author of the present study was, "I told this person about my thoughts and feelings." A total of eleven items measured attentiveness. Four items are from the CSI (Hess et al., 2007). A sample item 
from the CSI (Hess et al., 2007) for attentiveness was, "I paid attention to what feelings or emotions this person was experiencing within their messages." Seven of the items were created by the author for this study. A sample item for attentiveness created by the author of the current study was, "I tried to monitor the other person's feelings."

\section{Data Analysis}

\section{Factor analysis}

In order to assess the unidimensional nature of the openness and attentiveness variables, a series of exploratory factor analyses was performed. This analysis was also performed to check measurement validity and assess the potential for item reduction. Principal components analysis followed by oblique rotation was conducted four times. One was performed for openness for those who met offline as well as one was performed for openness for those who did not meet offline. One was also performed for attentiveness for those who met offline as well as one was performed for attentiveness for those who did not meet offline. Component extraction was based on a combination of eigenvalues greater than one and an inspection of scree plots.

For all four analyses, unidimensional scales were deemed most interpretable after inspecting the unrotated component loadings as well as loadings on rotated factors. The loading criteria were at least .60 for primary loading and no higher than .40 for crossloading. The vast majority of items in all conditions landed on the first unrotated component. Second and third rotated components loaded three or fewer items.

\section{Reliability}

A reliability analysis was performed on each of the groups of items for both the participants who met someone offline and those who did not. The first set of nine 
openness items for those who had met someone offline had a weak reliability (Cronbach's $\alpha=.64)$. Once item six was removed the alpha improved (Cronbach's $\alpha=$ .75). The scale with eight items had an acceptable reliability. Next, the author measured the set of 11 attentiveness items for those who had met someone offline which lead to a reliable scale (Cronbach's $\alpha=.79$ ). When item eight was removed the reliability increased (Cronbach's $\alpha=.82)$.

These same tests were then run on the groups who had not met someone offline. The first analysis was the nine items for openness that had a reliable scale (Cronbach's $\alpha$ $=.73$ ). Once again, when item six was removed the reliability increased (Cronbach's $\alpha=$ .83). This was then the same scale of eight items used for the group who met someone offline. The second analysis was on the 11 items for attentiveness which had a reliable scale (Cronbach's $\alpha=.92$ ). To stay consistent with the scale used to measure the participants who met someone offline and those who did not, item eight was also removed from this scale as well which slightly raised the reliability (Cronbach's $\alpha=.93$ ). Thus, an eight item scale and a 10 item scale were used to measure openness and attentiveness, respectively, both for those who met someone offline and those who did not. 


\section{CHAPTER IV}

\section{RESULTS}

In order to test $\mathrm{H}_{1}$ and $\mathrm{H}_{3}$, paired-sample $t$-tests were computed to compare levels of openness and attentiveness for the two groups: one where a participant met someone offline, and one where they did not. The sample that was used contained only those who had answered both parts of the survey. Results indicated that there is a significant difference in the level of openness when comparing these experiences. This was also true for the level of attentiveness. The analysis for openness produced a significant $t$ value with a strong effect size $(t(116)=7.331, p<.001, r=.56)$. An examination of the means showed that those who met another person offline $(M=4.91, S D=0.91)$ reported a higher amount of openness than with the parties they did not meet offline $(M=4.0, S D=$ 1.13), which supports $\mathrm{H}_{1}$. The analysis for attentiveness produced a significant $t$ value with a strong effect size $(t(116)=8.022, p<.001, r=.60)$. An examination of the means showed that those who met another person offline $(M=5.96, S D=0.71)$ reported a higher amount of attentiveness than with the parties they did not meet offline $(M=5.09$, $S D=1.23)$, which supports $\mathrm{H}_{3}$.

Correlation coefficients were computed to examine the relationship between the time it took for participants to meet the person they met offline from the first message exchanged and the amounts of openness and attentiveness. Time was measured in days. Only participants who took the first part of the survey were utilized for these tests because they were the ones who had met someone offline. The correlation between the 
amount of openness and the time it took for couples to move offline was positive, weakly correlated, and statistically significant $(r=.21, p=.003)$, contrary to $\mathrm{H}_{2}$. The correlation between the amount of attentiveness and the time it took for couples to move offline was positive, negligibly correlated, and not significantly significant $(r=.10, p=.090)$ and did not support $\mathrm{H}_{4}$. The correlation between openness and attentiveness was positive, moderately correlated, and statistically significant $(r=.51, p<.001)$.

To examine $\mathrm{H}_{5}$, a chi-square test was performed to examine whether men initiated to move to meet offline more often than women. The analysis produced a significant $\chi^{2}$ (29.30, $d f=1, p<.001)$. Regardless of whether a male or female was reporting, more men $(75.4 \%)$ were reported as asking to meet offline first compared to women (24.5\%). Further, out of the 43 males reporting who asked to move offline first, 33 (76.7\%) reported being the first to ask. Out of 75 females reporting who asked to move offline, 56 (74.7\%) reported the other party asked first. This indicates that men were more likely to ask to move offline, which supports $\mathrm{H}_{5}$. Same sex couples were excluded from this part of the analysis. 


\section{CHAPTER V DISCUSSION}

The purpose of this study was to examine the communicative strategies that are utilized to move an online relationship to a FtF meeting, which is considered a successful use of the online dating medium. In traditional dating, liking is a major variable that helps determine whether a couple continues to date. Also, openness and attentiveness are two of the most pertinent factors that lead to liking. This study found that there are higher amounts of both of these variables reported from online daters who met someone offline compared to when they reported on a person who they did not meet offline.

The results suggest that the strategies used offline in traditional dating relationships apply to the new online medium as well. Finkel et al. (2012) argued that online dating has fundamentally altered the acquaintance process for dating, mostly due to the abundant, available information on the other person. Although there are certainly some differences with the way courting is conducted online, this study suggests the idea that some parts of online dating share similarities with traditional offline dating.

The results of the current study show that some of the most effective strategies that enhance liking in a developing offline relationship, namely openness and attentiveness (Clark et al., 2009; Hess et al., 2007), are found in greater amounts for couples in online relationships who met offline compared to those who did not meet offline. These results suggest that in the same way these strategies aid in offline 
relationship development, they are improtant to utiltilize to find success in developing online relationships as well. This study also supports the idea that, just like in traditional dating situations, not utilizing these strategies decreases one's chance of a second meeting, or in this case an offline meeting, because without liking, future relationships are doomed (Rubin, 1973). The point of online dating is to meet other people. In order to accomplish this, the present author suggests one must employ these two strategies to achieve success.

The statistical significance and the size of the effects add to the results found in this study. With strong results in both of these areas, the conclusions drawn are more noteworthy. There are obviously more variables in effect that lead to a FtF meeting, but these results help shed some light on a two of the variables that are at work. It also shows that these two variables have a strong influence on moving offline. Before this study, it was unknown what variables were at play during the exchange-of-messages phase of online dating, however now we have a good base to build from with attentiveness and openness.

One aspect not shown by this study is whether or not the meeting will lead to a romantic relationship. There are many things that come into play once the two people meet that cannot be controlled for during the message-exchange phase of online dating. Perhaps once a meeting occurs, the person does not meet the idealized version created from the online interactions, as explained by the hyperpersonal model (Walther, 1996). Perhaps something does not click, or feel right, or there is no chemistry between the two would-be partners. Once the couple meets, the relationship goes beyond the scope of this study. So, while openness and attentiveness appear to contribute to a FtF meeting, the 
results from this study do not predict whether or not the meeting will progress beyond that phase of online dating.

Originally, the present author predicted that being more open and attentive to the other party online would lead to an expedient move to a FtF meeting. The results proved to show this is not the case. With openness, there is actually a positive relationship with the time it takes couples to move offline after the first message exchange, albeit a small one. So, if couples waited longer before moving offline, the amount of openness increased. When looking at the relationship between time before moving offline and openness in this light, the relationship makes sense. Of course the longer one communicates with another person, the more open about themselves they can become. If not related to speed of moving offline, there still must be some threshold of openness whereby there is enough openness to generate liking and a desire to move offline and learn more FtF. If there is a low amount of liking in an initial interaction, it is likely the couple does not continue messaging back and forth, let alone meet offline.

The relationship between attentiveness the time it took for a couple to move offline after the first exchanged message was not significant. The correlations of both attentiveness and openness with time to move offline are small, so it is likely there are other more important variables in play that affect speed of moving offline. Perhaps people's past experiences with online dating has a stronger relationship with how quickly they are willing to move offline. If they have had positive experiences meeting people, maybe they wish to skip the slow relationship building during message exchanges and put more weight in the $\mathrm{FtF}$ meeting. Another thought might be how comfortable they are in social settings. If a person is more extroverted, perhaps they prefer to get to know 
someone in person as opposed to building up liking and rapport online. Perhaps suggestion of a meeting place plays a role as well in that a public place may be safer or less intimidating than a dinner date. With both openness and attentiveness, it seems the influence on whether or not a couple moves offline is more affected than how soon that meeting happens.

This study attempted to examine a linear relationship for both attentiveness and openness with the speed it takes to move offline. There is a possibility that there is a curvilinear relationship where too little or too much of either variable will be detrimental to moving to a FtF meeting. In this scenario there would be certain levels in between the extremes of either variable that would be best for moving offline with haste. This study has the assumption that an online dater's goals are to meet the other person FtF as soon as possible, but it is certainly possible that she or he prefers the casual online interaction to a quicker meeting with a new person, which could influence the speed at which they want to move offline.

The results of this study also show that men are much more likely to propose a FtF meeting than are women. This makes sense because men tend to send more messages online (Hitsch et al., 2010). In traditional dating, men are typically expected to ask women out as well, so it follows that they would also be expected to in online dating, since the first FtF meeting is comparable to a "first date." Another factor that lends to this conclusion is that women tend to be more passive in dating environments (Clark et al., 1999) and therefore may be passive in asking to meet during online interactions. Women also are the gatekeepers when it comes to allowing a sexual relationship (Cunningham, 1989) and may be more reluctant than men to move offline quickly. 


\section{Limitations}

There are some limitations to the study. The first limitation was that there could have been a better way to compare groups who took the survey. A variable was not created to separate and compare groups who had either only met someone offline and those who had not met someone offline. A different result might come from comparing independent samples that had one experience or the other, as compared to examining a paired group that had both experiences. The positive experience of meeting someone compared to the experience where they did not meet someone could also have an effect on how participants responded to the survey in each section. This could also be controlled in future replications by reversing the order in which some participants answered the two sections of the survey, which controls the order effect.

Some of the demographics were designed to be captured only after the second section of the survey with the assumption that those who had met someone offline would also complete the second part of the survey. This, unfortunately, did not occur for everyone as many participants only completed the first part of the survey. For example, $37.7 \%$ of participants did not report their sex because of this flaw in the design of the survey. This flaw also affected participants reporting their experience with online dating, time since their interaction, and how often they checked their online dating site. In future renditions, there should be a screening question after each section that either moves them to the next section, if applicable, or directly to a demographics section.

Another limitation is how the sample was obtained. In a perfect world, a sample could have been obtained directly from an online dating service as opposed to posting an online survey link, open for everyone. Posting on Reddit and through the author's 
Facebook leads to a more homogenous sample and limits the generalizability of the data. Similar to this, the majority of the sample was Caucasian, and once again this decreases generalizability.

There is also a self-selection bias that accompanies online surveys in that in a given online community there are certain types of participants who are more likely to answer online surveys (Wright, 2006). This may be especially relevant to the use of Reddit's r/samplesize, which is a community where surveys are submitted and taken, which is the purpose of the online community. These members could be seen as expert survey takers, since that is what the community is geared towards. They also may not represent the general population of online daters. Something else to consider is that the participants who use Reddit are used to interacting with others online. This experience may influence how they interact on other online places such as online dating sites. The sample in the current study is not representative of all who utilize online dating sites. In other words, Reddit users can be seen as experts of the medium. These factors can limit the generalizability of the results.

\section{Future Directions}

Many participants reported that more than a year had passed since their interaction, which can influence responses to the survey. For example, if the person they met offline became a steady relationship, the participant may report higher levels of openness and attentiveness for the first part of the survey, even if at the time the actual levels were lower. This would be because the positive feelings they derive from the person now may be reported instead of the way they actually felt during their first interactions. One way to improve results in future studies would be to survey those who 
have had an experience recently, perhaps even right after their first FtF meeting. This would ensure the reported results would be more accurate because less time would pass between the meeting and reporting the results. This would help eliminate the recall bias.

One difficulty with researching only one step of the online dating process is that there may be some carryover from previous steps in online dating that will influence whether or not a couple will move offline. For instance, physical attraction likely plays a larger role in meeting someone offline. It certainly factors into whether a person will exchange messages in the first place. Perhaps if the physical attraction is great enough, a lack of openness and attentiveness can be overcome and the pair will still meet offline. Researchers could also examine whether or not this study's variables influence the quality of the FtF meeting or perhaps if they related to a relationship forming postmeeting. It could be that if partners are more open and attentive online, there is a better first meeting because the pair already feels more comfortable with the other person and feels less like she or he is meeting a stranger.

Future studies should examine not only which strategies enhance liking but also those that decrease liking. An example of this could be dating goals such as a person searching only for a hook up. Even if an online dater is open about this goal, this could certainly conflict with the other person's goals and prevent an offline meeting. Once enough interaction variables have been examined, the process of online dating could be streamlined and aid those who wish to utilize this medium for courting. This can also determine if any of these variables or others magnify or diminish the effects of openness and attentiveness. Future studies could include a scale that directly measures liking, as opposed to variables that presumably lead to liking, as was done in this study. 
Future studies should examine more about the structure of communication that occurs during this step in online dating. For example, a future study may consider examining the same variables of openness and attentiveness in the other person as well. These studies may find that there is a big influence between what the other person does with his or her communication and the outcome of the interaction. The design of the current study only considers half of what is occuring during this process because we only see one side of the communication. It is certainly possible that if the other person is not utilizing these strategies as well, that there will not be an offline meeting. It would be important to study both sides of the process.

Something else future studies should examine is how these two variables, openness and attentiveness, relate to other types of CMC, such as video gaming and forum websites. This study lends to the idea that meaningful relationships can be formed online, especially if traditional tactics are employed. Because of technology advances, more and more human interaction will be done via CMC. So, understanding these relationships is integral to the future of communication. Future studies should also examine relationship formations in different types of online relationships, such as work and friendship relationships, and whether or not the variables in this study aid in forming these other types of relationships in CMC environments. For example, these strategies might be useful in work relationships that use a majority of telecommuting and some eventual FtF meetings in that with a higher level of liking there is better interaction between these co-workers.

Before this study, there was not much research on what occurred between people who utilized online dating during their message exchange that lead to a FtF meeting. This 
study sheds some light on two of the variables that are present during this exchange. The results here are only a start to understanding what occurs during that message exchange that determines whether someone continues exchanging messages and eventually moves offline or whether they cease communication. These variables are integral to the entire process of online dating.

We as a society have become more and more accepting of online dating as a viable way to meet our significant other. This trend looks to continue into the future. It seems that as new technology arrives we use it in new ways to find potential partners. The more we understand about online dating now will aid in utilizing these new technologies as they emerge. 


\section{REFERENCES}

Ahuvia, A. C., \& Adelman, M. B. (1992). Formal intermediaries in the marriage market: A typology and review. Journal of Marriage and the Family, 54 452-463. doi: $10.2307 / 353076$

Altman, I., \& Taylor, D. A. (1973). Social penetration: The development of interpersonal relationships. New York, NY: Holt, Rinehart, \& Winston.

Baker, A. J. (2005). Double click: Romance and commitment among online couples. Cresskill, NJ: Hampton Press.

Bell, R. A., \& Daly, J. A. (1984). The affinity-seeking function of communication. Communications Monographs, 51, 91-115. doi: 10.1080/03637758409390188

Bredow, C. A., Cate, R. M., \& Huston, T. L. (2008). Have we met before? A conceptual model of first romantic encounters. In S. Sprecher, A. Wenzel, \& J. Harvey (Eds.), Handbook of relationship initiation (pp. 3-28) New York, NY: Psychology Press.

Cegala, D. J. (1981). Interaction involvement: A cognitive dimension of communicative competence. Communication Education, 30, 109-121. doi: $10.1080 / 03634528109378460$

Cegala, D. J. (1984). Affective and cognitive manifestations of interaction involvement during unstructured and competitive interactions. Communications Monographs, 51, 320-338. doi: 10.1080/03637758409390205

Cegala, D. J., Savage, G. T., Brunner, C. C., \& Conrad, A. B. (1982). An elaboration of the meaning of interaction involvement: Toward the development of a theoretical concept. Communications Monographs, 49, 229-248. doi: $10.1080 / 03637758209376087$

Clark, C. L., Shaver, P. R., \& Abrahams, M. F. (1999). Srategic behaviors in romantic relationship initiation. Personality and Social Psychology Bulletin, 25, 709-722. doi: $10.1177 / 0146167299025006006$

Collins, N. L., \& Miller, L. C. (1994). Self-disclosure and liking: A meta-analytic review. Psychological Bulletin, 116, 457-475. doi: 10.1037/0033-2909.116.3.457 
Cunningham, M. R. (1989). Reactions to heterosexual opening gambits: Female selectivity and male responsiveness. Personality and Social Psychology Bulletin, 15(1), 27-41. doi: 10.1177/0146167289151003

Derlega, V. J., Metts, S., Petronio, S., \& Margulis, S. T. (1993). Self-disclosure. Newbury Park, CA: Sage.

Ellin, A. (2009, Feb 11). The recession, isn't it romantic?. New York Times, p. 9.

Ellison, N., Heino, R., \& Gibbs, J. (2006). Managing impressions online: Selfpresentation processes in the online dating environment. Journal of ComputerMediated Communication, 11, 415-441. doi: 10.1111/j.1083-6101.2006.00020.x

Falk, D. R., \& Wagner, P. N. (1985). Intimacy of self-disclosure and response processes as factors affecting the development of interpersonal relationships. The Journal of Social Psychology, 125, 557-570. doi: 10.1080/00224545.1985.9712029

Finkel, E. J., Eastwick, P. W., Karney, B. R., Reis, H. T., \& Sprecher, S. (2012). Online dating: A critical analysis from the perspective of psychological science.

Psychological Science in the Public Interest, 13(1), 3-66. doi: $10.1177 / 1529100612436522$

Fiore, A. T., Taylor, L. S., Mendelsohn, G. A., \& Hearst, M. (2008, April). Assessing attractiveness in online dating profiles. In Proceedings of the twenty-sixth annual SIGCHI conference on human factors in computing systems (pp. 797-806). New York, NY: ACM. doi: 10.1145/1357054.1357181

Gardner, A. (2012, February 6). Psychologists highlight pitfalls of online dating. Retrieved from http://www.cnn.com/2012/02/06/health/online-datingpitfalls/index.html

Gibbs, J. L., Ellison, N. B., \& Heino, R. D. (2006). Self-presentation in online personals: The role of anticipated future interaction, self-disclosure, and perceived success in internet dating. Communication Research, 33, 152-177. doi: $10.1177 / 0093650205285368$

Gibbs, J. L., Ellison, N. B., \& Lai, C. H. (2011). First comes love, then comes Google: An investigation of uncertainty reduction strategies and self-disclosure in online dating. Communication Research, 38, 70-100. doi:10.1177/0093650210377091

Guadagno, R. E., Okdie, B. M., \& Kruse, S. A. (2012). Dating deception: Gender, online dating, and exaggerated self-presentation. Computers in Human Behavior, 28(2), 642-647. doi: 10.1016/j.chb.2011.11.010 
Heino, R. D., Ellison, N. B., \& Gibbs, J. L. (2010). Relationshopping: Investigating the market metaphor in online dating. Journal of Social and Personal Relationships, 27, 427-447. doi: 10.1177/0265407510361614

Henderson, S., \& Gilding, M. (2004). "I've never clicked this much with anyone in my life": Trust and hyperpersonal communication in online friendship. New Media \& Society, 6, 487-506. doi:10.1177/146144804044331

Henry-Waring, M., \& Barraket, J. (2008). Dating and intimacy in the 21st century: The use of online dating sites in Australia. International Journal of Emerging Technologies and Society, 6(1), 14-33. Retrieved from http://www.swin.edu.au/ijets

Hendrick, S., \& Hendrick, C. (1992). Liking, loving, and relating. ( $2^{\text {nd }}$ ed.). Belmont, CA: Brooks/Cole.

Hess, J. A., Fannin, A. D., \& Pollom, L. H. (2007). Creating closeness: Discerning and measuring strategies for fostering closer relationships. Personal Relationships, 14(1), 25-44. doi: 10.1111/j.1475-6811.2006.00140.x

Hitsch, G. J., Hortaçsu, A., \& Ariely, D. (2010). What makes you click?: Mate preferences in online dating. Quantitative Marketing and Economics, 8, 393-427. doi: 10.1007/s11129-010-9088-6

Jourard, S. M. (1964). The transparent self: Self-disclosure and well-being. Princeton, NJ: Van Nostrand.

Jourard, S. M., \& Landsman, M. J. (1960). Cognition, cathexis, and the "dyadic effect" in men's self-disclosing behavior. Merrill-Palmer Quarterly of Behavior and Development, 6, 178-186. doi: 10.2307/23082776

Kang, T., \& Hoffman, L. H. (2011). Why would you decide to use an online dating site? Factors that lead to online dating. Communication Research Reports, 28(3), 205213. doi: $10.1080 / 08824096.2011 .566109$

Long, B. L. (2010). Scripts for online dating: A model and theory of online romantic relationship initiation (Unpublished doctoral dissertation). Bowling Green State University, $\mathrm{OH}$.

Madden, M., \& Lenhart, A. (2006, Mar 5). Online dating. Pew Internet \& American Life Project. Retrieved from http://www.pewinternet.org/Reports/2006/OnlineDating.aspx

McKenna, K. Y. A. (2007). A progressive affair: Online dating to real world mating. In M. T. Whitty, A. J. Baker, \& J. A. Inman (Eds.), Online matchmaking (pp. 112124). Hampshire, England: Palgrave Macmillan. 
McKenna, K. Y. A. (2008). MySpace or your place: Relationship initiation and development in the wire and wireless world. In S. Sprecher, A. Wenzel, \& J. Harvey (Eds.), Handbook of relationship initiation (pp. 235-248). New York, NY: Psychology Press.

Merkle, E. R., \& Richardson, R. A. (2000). Digital dating and virtual relating: Conceptualizing computer mediated romantic relationships. Family Relations, 49, 187-192. doi: 10.1111/j.1741-3729.2000.00187.x

Mitchell, R. L. (2009, Feb 13). Online dating: The technology behind the attraction. Retrieved from http://www.computerworld.com/s/article/9127711/Online_dating_The_technolog y_behind_the_attraction?intsrc=hm_ts_head

Newcomb, T. M. (1961). The acquaintance process. New York, NY: Holt, Rinehart and Winston

Norton, R. W., \& Pettegrew, L. S. (1979). Attentiveness as a style of communication: A structural analysis. Communications Monographs, 46, 13-26. doi: $10.1080 / 03637757909375987$

O'Sullivan, B. (2000). What you don't know won't hurt me. Human Communication Research, 26, 403-431. doi: 10.1111/j.1468-2958.2000.tb00763.x

Orenstein, S. (2003, Aug 1). The love algorithm match.com thinks it has found a formula for ruling the fiercely competitive online dating business: A scientific way to find Mr. or Ms. right. Retrieved from http://money.cnn.com/magazines/business2/business2_archive/ 2003/08/01/346313/index.htm

Pinel, E. C., Long, A. E., Landau, M. J., Alexander, K., \& Pyszczynski, T. (2006). Seeing I to I: A pathway to interpersonal connectedness. Journal of Personality and Social Psychology, 90, 243-257. doi: 10.1037/0022-3514.90.2.243

Rabby, M. K. (2007). Relational maintenance and the influence of commitment in online and offline relationships. Communication Studies, 58, 315-337. doi: $10.1080 / 10510970701518405$

Ramirez Jr., A., \& Zhang, S. (2007). When online meets offline: The effect of modality switching on relational communication. Communication Monographs, 74, 287310. doi: $10.1080 / 03637750701543493$

Rice, R. E., \& Love, G. (1987). Electronic emotion socioemotional content in a computer-mediated communication network. Communication Research, 14, 85108 . 
Rubin, Z. (1973). Liking and loving: An invitation to social psychology. New York, NY: Holt, Rinehart and Winston.

Rubin, R. B., \& Martin, M. M. (1994). Development of a measure of interpersonal communication competence. Communication Research Reports, 11, 33-44. doi: $10.1080 / 08824099409359938$

Sanderson, C. A., Keiter, E. J., Miles, M. G., \& Yopyk, D. J. A. (2007). The association between intimacy goals and plans for initiating dating relationships. Personal Relationships, 14, 225-243. doi: 10.1111/j.1475-6811.2007.00152.x

Smith, A., \& Duggan, M. (2013, Oct. 21). Online dating \& relationships. Retrieved from http://pewinternet.org/Reports/2013/Online-Dating.aspx

Spitzberg, B. H., \& Dillard, J. P. (2002). Social skills and communication. In M. Allen, R. W. Preiss, B. M. Gayle, \& N. Burrell (Eds.), Interpersonal communication research: Advances through meta-analysis (pp. 89-107). Mahwah, NJ: Lawrence Erlbaum Associates

Sprecher, S. (2011). Internet matching services: The good, the bad, and the ugly (disguised as attractive). In W. Cupach \& B. Spitzberg (Eds.), The dark side of close relationships II (pp. 119-143). New York, NY: Routledge.

Sprecher, S., \& Metts, S. (2013) Logging on, hooking up: The changing nature of romantic relationship initiation and romantic relating. In C. Hazan \& M. I. Campa (Eds.), Human bonding: The science of affectionate ties (pp. 197-225). New York, NY: Guilford Press.

Sprecher, S., Treger, S., \& Wondra, J. D. (2013). Effects of self-disclosure role on liking, closeness, and other impressions in get-acquainted interactions. Journal of Social and Personal Relationships, 30, 497-514. doi:10.1177/0265407512459033

Valkenburg, P. M., \& Peter, J. (2007). Who visits online dating sites?: Exploring some characteristics of online daters. CyberPsychology \& Behavior, 10, 849-852. doi: $10.1089 / \mathrm{cpb} .2007 .9941$

Walther, J. B. (1992). Interpersonal effects in computer-mediated interaction: A relational perspective. Communication Research, 19, 52-90. doi: $10.1177 / 009365092019001003$

Walther, J. B. (1996). Computer-mediated communication: Impersonal, interpersonal, and hyperpersonal interaction. Communication Research, 23, 3-43. doi: $10.1177 / 009365096023001001$

Walther, J. B. (2007). Selective self-presentation in computer-mediated communication: Hyperpersonal dimensions of technology, language, and cognition. Computers in Human Behavior, 23, 2538-2557. doi: 10.1016/j.chb.2006.05.002 
Whitty, M. T. (2008). Revealing the "real" me, searching for the "actual" you: Presentations of self on an internet dating site. Computers in Human Behavior, 24, 1707-1723. doi: 10.1016/j.chb.2007.07.002

Whitty, M. T., \& Carr, A. N. (2006). Cyberspace romance: The psychology of online relationships. Hampshire, UK: Palgrave Macmillan.

Wright, K. B. (2006). Researching Internet-based populations: Advantages and disadvantages of online survey research, online questionnaire authoring software packages, and web survey services. Journal of Computer-Mediated Communication, 10(3). doi: 10.1111/j.1083-6101.2005.tb00259.x

Wysocki, D. K. (1998). Let your fingers do the talking: Sex on an adult chat-line. Sexualities, 1, 425-452. doi: 10.1177/136346098001004003 


\section{APPENDIX}

\section{SURVEY QUESTIONNAIRE}

Have you ever exchanged messages with anyone on an online dating site (OKcupid, match.com, eHarmony, Plenty of fish, Zoosk, SinglesNet, or many others) THAT YOU EVENTUALLY MET OFFLINE? Yes or No (If no auto-redirect to the second part of the survey)

When taking this portion of the survey, think about a person you have interacted with an online dating site such as OKcupid, match.com, eHarmony, Plenty of fish, Zoosk, SinglesNet, or many others, that you decided to meet offline or face to face. Be sure this is someone that you have exchanged messages with on the website or that you have exchanged text or phone conversations with and then decided to move offline. When answering the questions be sure that your answers reflect how you communicated during this exchange of messages online BEFORE you met offline and not after you met the person. $(\mathrm{O}=$ Openness; $\mathrm{A}=$ Attentiveness. * indicates reversed polarity question. + indicates item that was removed for final scale)

Participants will choose to what degree the agree or disagree with each following statement with the following scale:

1-->Strongly Disagree

2--> Moderately Disagree

3--> Slightly Disagree

4--> Neither Disagree or Agree

5--> Slightly Agree

6--> Moderately Agree

7--> Strongly Agree

1. I was willing to discuss topics with this person that I wouldn't normally talk about with others online. [Hess et al., 2007; O]

2. I was more talkative with this person than I might be with someone else online. [Hess et al., 2007; O] 
3. I told this person information about myself that was more personal than what I would share with just anyone online. [Hess et al., 2007; O]

4. I tried not to forget things that this person had told me within their messages. [Hess et al., 2007; A]

5. I treated this person as someone who was worthy of respect and dignity. [Hess et al., 2007; A]

6. I paid attention to what feelings or emotions this person was experiencing within their messages. [Hess et al., 2007; A]

7. I paid careful attention when this person was writing to me. [Hess et al., 2007; A]

8. I told this person about my thoughts and feelings. [O]

9. I was open with this person with my messages. [O]

10. I put a lot of thought into constructing the messages that I sent to this person. [A]

11. I made references to past conversation topics that this person and I had previously discussed [A]

12. This person was honest with me.

*13. I did not trust this person.

14. I was looking for long-term relationships when using online dating sites when I first contacted this person.

*15. I did not use online dating sites to search for long-term relationships when I first contacted this person.

*16. I was reluctant to disclose information about myself to this person. [O]

17. I was able to successfully convey my personality to this person. [O]

*18. The other person did not get an accurate picture of who I am. [O]

*19. When responding to this person's messages I did not put much thought into how I 
should respond. [A]

+20. I adapted my messages to what was appropriate for this person. [A]

*21. I did not show respect to the other person in my messages. [A]

*22. I did not find the content of this person's messages important. [A]

23. I tried to monitor the other person's feelings [A]

$*+24$. I was guarded in what I was willing to tell the other person. [O]

What is the length of time, in days, you waited to meet this person face to face after first sending an online message to this person

Who first asked to meet offline? I did The other person did

Did you continue to date the person after the first face to face meeting? Yes No___ If so, how long did/has it last/lasted in weeks?

When taking this portion of the survey, think about a person you have interacted with an online dating site such as OKcupid, match.com, eHarmony, Plenty of fish, Zoosk, SinglesNet, or many others, that you decided NOT to meet offline or face to face. Be sure this is someone that you have exchanged messages with on the website or that you have exchanged text or phone conversations with. When answering the questions be sure that your answers reflect how you communicated during this exchange of messages and not after you decided to cease communication with the person. $(\mathrm{O}=$ Openness; $\mathrm{A}=$ Attentiveness)

Participants will choose to what degree the agree or disagree with each statement with the following scale:

1-->Strongly Disagree

2--> Moderately Disagree

3--> Slightly Disagree

4--> Neither Disagree or Agree

5--> Slightly Agree

6-- Moderately Agree

7--> Strongly Agree 
1. I was willing to discuss topics with this person that I wouldn't normally talk about with others online. [Hess et al., 2007; O]

2. I was more talkative with this person than I might be with someone else online. [Hess et al., 2007; O]

3. I told this person information about myself that was more personal than what I would share with just anyone online. [Hess et al., 2007; O]

4. I tried not to forget things that this person had told me within their messages. [Hess et al., 2007; A]

5. I treated this person as someone who was worthy of respect and dignity. [Hess et al., 2007; A]

6. I paid attention to what feelings or emotions this person was experiencing within their messages. [Hess et al., 2007; A]

7. I paid careful attention when this person was writing to me. [Hess et al., 2007; A]

8. I told this person about my thoughts and feelings. [O]

9. I was open with this person with my messages. [O]

10. I put a lot of thought into constructing the messages that I sent to this person. [A]

11. I made references to past conversation topics that this person and I had previously discussed [A]

12. This person was honest with me.

*13. I did not trust this person.

14. I was looking for long-term relationships when using online dating sites when I first contacted this person.

*15. I did not use online dating sites to search for long-term relationships when I first contacted this person.

*16. I was reluctant to disclose information about myself to this person. [O] 
17. I was able to successfully convey my personality to this person. [O]

*18. The other person did not get an accurate picture of who I am. [O]

*19. When responding to this person's messages I did not put much thought into how I should respond. [A]

+20. I adapted my messages to what was appropriate for this person. [A]

*21. I did not show respect to the other person in my messages. [A]

*22. I did not find the content of this person's messages important. [A]

23. I tried to monitor the other person's feelings [A]

$*+24$. I was guarded in what I was willing to tell the other person. [O]

(The following questions will be asked after both survey group)

How long ago did this interaction with the other person take place? Less than a month ago 1-6 months ago 6-12 months ago More than 12 months ago

During the interaction online with the other person I would check my online dating site More than once a day About once a day Every couple of days About once a week Less than once a week

How long have you used online dating sites? Less than a month $1-6$ months 6-12 months More than 12 months

What dating sites have you used? (Check all that apply) Okcupid eHarmony Zoosk SinglesNet Plenty of Fish Match

Your Age? Years

Age of the other person (according to the profile)? Years

Your Sex? Male Female Sex of the other person? Male Your race? Caucasian Female

Latino Pacific Islander African American Asian disclose Other Prefer not to 